\title{
Flattop Beam Generation and Multibeam Processing Using Aspheric and Diffractive Optics
}

\author{
Keiji FUSE \\ Sumitomo Electric Hardmetal Corp., 1-1-1, Koya-kita, Itami, Hyogo, 664-0016 Japan \\ E-mail: fuse-keiji@sei.co.jp
}

\begin{abstract}
State-of-the-art optical techniques have been employed in the recent development of laser materials processing technology. One advanced application of aspheric optics is in homogenization, i.e., flattop beam generation, where an aspheric lens directly controls the wavefront of a laser beam and transforms its Gaussian intensity distribution into one with a circular flat top. Diffractive optics also function as a homogenizer, giving more flexibility in beam shapes, including square, rectangular, linear, or other complex shapes depending on the application. Those aspheric and diffractive homogenizers can be utilized for laser drilling, scribing, annealing, etc. Another important function of diffractive optics is in beam splitting for multibeam processing. A single laser beam is split into multiple beams that are converged onto multiple spot positions on a workpiece by a special lens having an f-sin $\theta$ distortion characteristic. Since multibeam processing enables higher throughput with a simpler optical system than the conventional one, diffractive beam splitters are widely used for the laser processes in the mass production of electronic devices. In cases where a flattop multibeam is required, a homogenizer can be installed in the multibeam optical system. In this report, some examples of optical systems for practical applications are presented, with their design concepts and specifications.
\end{abstract}

DOI:10.2961/jlmn.2010.02.0011

Keywords: aspheric, diffractive optics, homogenizer, flattop beam, multibeam, laser processing

\section{Introduction}

Recent developments in laser technology have widened the variety of selection for high-power lasers, and it has therefore become much more common to employ laser materials processing at actual production sites. Advances in compact, high-power, and highly stable single mode lasers, such as diode-pumped solid state lasers and fiber lasers are making a great contribution, and because of this, high quality laser beams can now be utilized more easily than before. In addition, choices in wavelength between infrared, visible, and ultraviolet lasers are available to users to suit the application. In some of the latest applications, users themselves have developed their own original processes and equipment to suit their purpose in order to obtain a higher level of performance and larger cost reductions that cannot be achieved by any competitive process. In the electronics industry, for example, microvia drilling of multilayer printed wiring boards and low-temperature polysilicon annealing are used in the mass production of electronic devices and equipment, including cell phones, notebook computers, and flat panel displays (liquid crystal displays and plasma display panels). Applying advanced laser processing has finally characterized the performance and functions of the products.

In the innovative applications of laser processing described above, optics play an important role as well as laser oscillators. Although every laser processing machine has lenses and mirrors, it should be mentioned that almost all newly-developed applications employ advanced laser optics as the key device, which characterizes the advantages of the processing. Aspheric lenses and diffractive optical elements (DOEs) are typical examples of this advanced optics. Aberration correction by aspheric optics has already become the standard way to obtain a diffraction-limited spot size for a laser beam, including flat field telecentric $\mathrm{f} \theta$ lenses for PWB laser drilling and aberration-corrected lenses for ultraviolet laser processing. Flattop generation is an advanced application of both aspheric and diffractive optics. These optical components transform a Gaussian beam into circular, rectangular or other variously-shaped flattop beams. DOEs are also used for beam splitting from a single incoming beam to multiple output beams. There is a recent trend for innovative laser processing machines to be equipped with an optical unit consisting of a train of multiple advanced optical elements, instead of introducing a single element on an existing machine. The unit is designed to function maximally as a unified optical system, consequently achieving a substantial reduction in time for optical adjustments and an upgrade to the machine's performance.

In this paper, the author describes aspheric optics, DOEs and optical units that have already been employed in concrete processing applications, or that will be employed in the near future, and in particular, flattop beam generation using beam homogenizers and multibeam processing using diffractive beam splitters.

\section{Flattop beam generation by homogenizers}

Since the uniform intensity distribution of a flattop beam is required instead of the Gaussian distribution of a single mode laser, surface treatment processes such as laser annealing utilize beam homogenization technologies including aspheric and diffractive optics [1, 2]. Integrating optics with lens array devices like a fly's eye lens is 


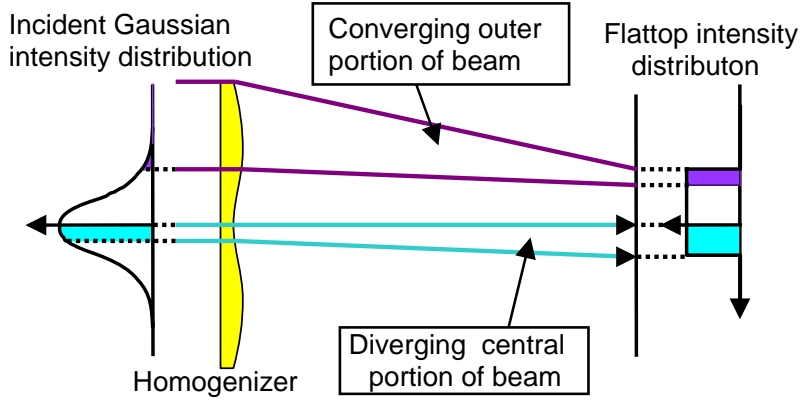

Fig. 1 Principle of flattop beam generation

another method of beam homogenization, which can be achieved by dividing a beam laterally on a plane perpendicular to the optical axis and by superposing the divided portions of the beam onto a focal point to average out variations in intensity. Although it can be used with a laser beam, it can cause a spiky and less uniform intensity distribution, because of the high degree of coherence of the laser beam. In contrast, both aspheric and diffractive homogenizers generate a flattop beam by diverging the higher intensity central portion of the incoming Gaussian beam and by converging the lower intensity outer portion, as shown in Fig. 1. Since no beams cross each other between the homogenizer and the focal plane, high uniformity is obtainable for the flattop beam (less than $\pm 10 \%$ ), even when the incoming beam is highly coherent. Diffractive homogenizers are superior to aspheric ones in the variety of flattop beam shapes they can generate, including shapes other than the above rectangle. Aspheric homogenizers can only generate circular flattops, but have efficiencies of $100 \%$ and no diffraction losses, while the diffraction efficiency of the DOE homogenizer is usually 80 to $95 \%$ due to diffraction losses.

Figure 2 shows an aspheric homogenizer that generates a $\phi 65 \mu \mathrm{m}$ diameter (1/ $\mathrm{e}^{2}$ width) flattop beam from a $\phi 10$ $\mathrm{mm}$ diameter ( $1 / \mathrm{e}^{2}$ width) single mode beam of the second harmonic generation (SHG) of YAG laser. The focal length of the homogenizer is $200 \mathrm{~mm}$. As shown in this figure, the beam propagates changing the intensity profile, and has a flattop profile just at the focal point $(Z=0)$ of the homogenizer. Before the focal point $(Z<0)$, the beam is larger with a rounder top profile. After the focus $(Z>0)$,

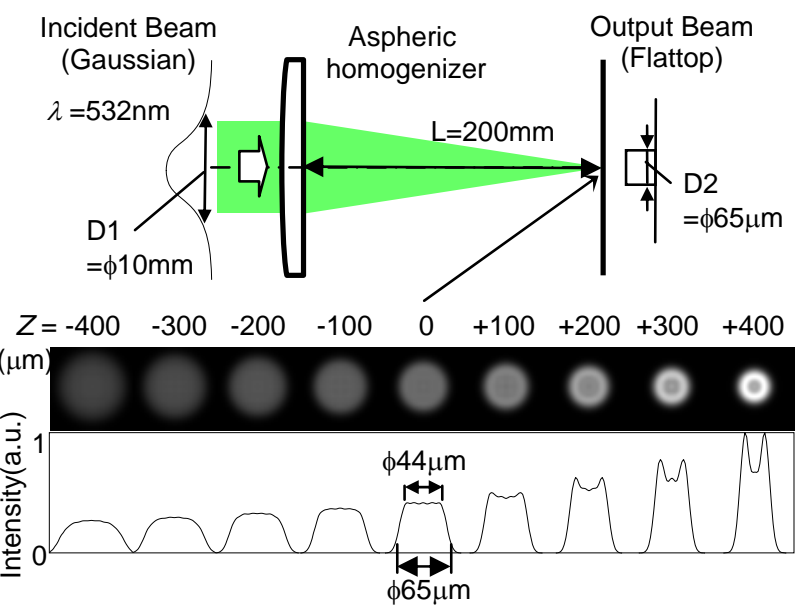

Fig. 2 Single element aspheric homogenizer the beam becomes smaller and makes a ringer spot. Further beam propagation makes the ringer spot smaller and finally concentrated to the central spot, which has the maximum peak intensity during propagation. Those intensity variations are caused by the wavefront errors of the beam, which is induced by the aspheric homogenizer to make a flattop spot. To reduce the intensity variations during propagation, the wavefront errors should be corrected by the second aspheric element. Figure 3 shows an example of the two-element type homogenizer that can output a collimated flattop beam. The first aspheric element (\#1) transforms a $\phi 8 \mathrm{~mm}\left(1 / \mathrm{e}^{2}\right.$ width) Gaussian $\mathrm{CO}_{2}$ laser beam to $\phi 8 \mathrm{~mm}$ diameter (top width) flattop beam at the focal position that is $150 \mathrm{~mm}$ distant from the \#1 element. The second element (\#2) placed at the focal position functions to collimate the flattop beam by correcting the distorted wavefront to be flat. Thus, the collimated flattop beam can propagate long distance with small intensity variations as shown in Fig. 3.

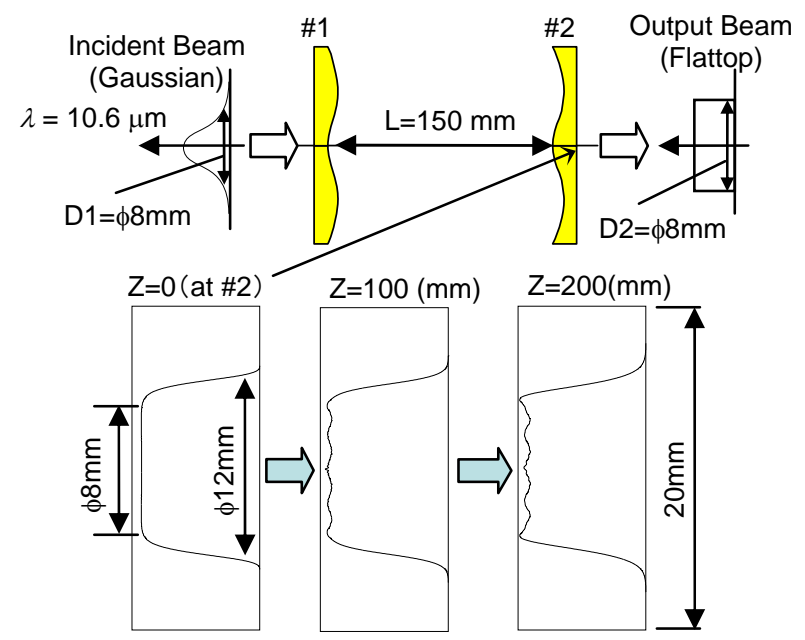

Fig. 3 Two-element type aspheric homogenizer

Figure 4 indicates a DOE homogenizer that has a 70 $\mathrm{mm}$ focal length and generates a $1.0 \mathrm{~mm} \times 0.5 \mathrm{~mm}$ rectangular flattop beam from a $\phi 2.0 \mathrm{~mm}$ diameter $\left(1 / \mathrm{e}^{2}\right.$ width) single mode SHG-YAG laser beam. There being various methods for optimizing the phase patterns of DOE homogenizers, the iterative Fourier transform algorithm (IFTA), which is one of the most practical optimization algorithms, is often applied in the design of DOE homogenizers, because in general a homogenizer has a large-scale phase pattern compared to the unit pattern of the periodic phase structure of the DOE beam splitter (described in the next section).

There are other applications requiring linear flattop beams instead of rectangular ones, including different kinds of thermal treatments covering large surface areas of a workpiece by scanning the line beam in a direction perpendicular to the long axis of the beam. Figure 5 shows the design result for a DOE homogenizer that transforms a $\phi 3.0 \mathrm{~mm}\left(1 / \mathrm{e}^{2}\right.$ width) single mode SHG-YAG laser beam into a 4-mm length linear flattop beam. The cross-sectional intensity profile on the shorter axis is a $0.1 \mathrm{~mm} 1 / \mathrm{e}^{2}$-width Gaussian distribution. As shown in this figure, it is obvious that the DOE homogenizer can achieve a uniform intensity 


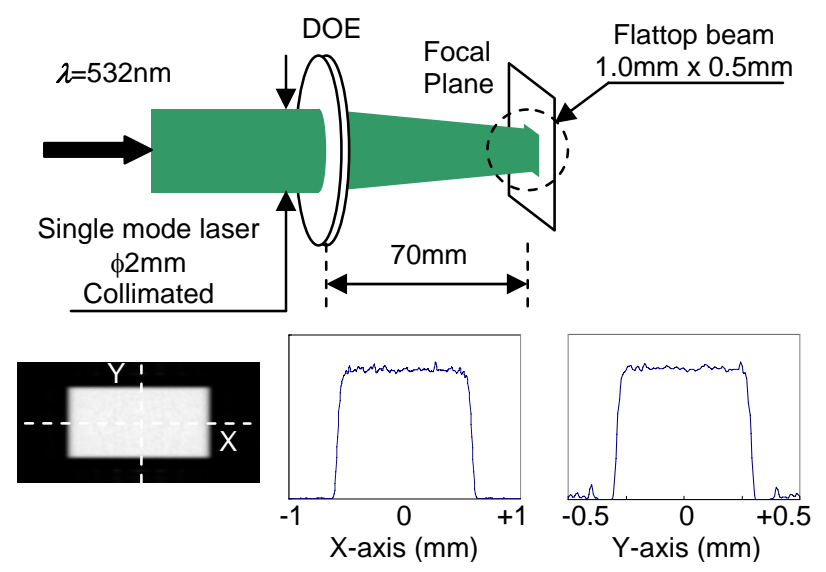

Fig. 4 Rectangular flattop beam generation

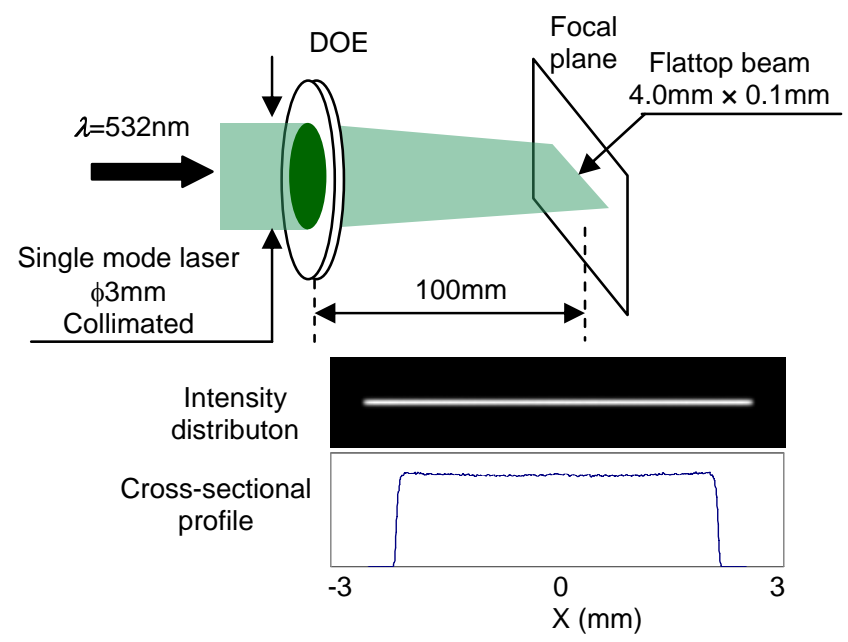

Fig. 5 Linear flattop beam generation

for the linear beam. An increase in the length of the line beam is required to increase productivity, and this creates difficulties due to a shortage of laser power. As shown in Fig. 6, a longer line beam with a high enough power/energy density could be achieved by connecting multiple linear beams in a straight line, each beam being generated by an individual laser oscillator. Each DOE is designed to match the properties of the corresponding laser beam. When the line beams are reconstructed to produce a single line on the focal plane, the edges of neighboring beams overlap. However, this overlap does not generally produce interference patterns, because the beams coming from

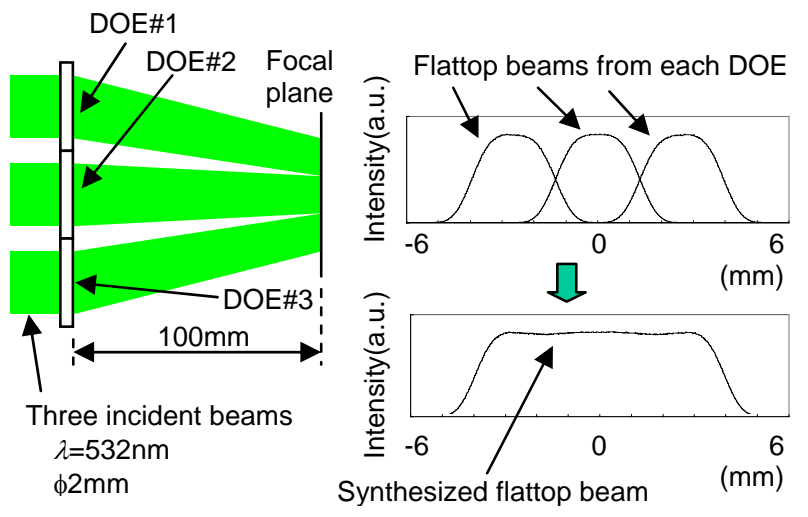

Fig. 6 Synthesis of long linear flattop beam independent lasers never interfere except for significantly short periods of time. The incoherence intensity summations for the line beam elements therefore provide the intensity distribution of the entire single line beam, each element of which is designed to have a specific profile so that when summed they produce a flat top. In Fig. 6, three beams are set to synthesize a $6-\mathrm{mm}$ linear beam. The synthesized beam has dull edges on both sides, because the beams are added to obtain a longer synthesized beam. The DOE homogenizers are independent, but it is possible to fabricate them simultaneously on a single substrate with accurate locations. Figure 7 shows the measurement results in a two beam synthesis experiment, indicating good agreement between the measured and the calculated intensity distributions.

It is known that the optical properties of aspheric and diffractive homogenizers are influenced by fabrication errors. Simulations calculated with the latest computer systems can give us good predictions for the performance degradation caused by various kinds of fabrication errors. However, it should be noted that homogenizers are more sensitive to variations in the incident beam than to fabrication errors. The conditions of the incident beam directly affect the uniform intensity profile of a flattop beam, because the design principle of the homogenizer is fundamentally a remapping of the incoming intensity distribution, as shown in Fig. 1. The uniformity of the flattop beam is degraded once the incoming beam deviates from the desired position, size or intensity distribution. Care with the laser mode quality and adjustment of the beam parameters is therefore essential. A beam expander is needed to adjust the beam diameter (zoom type is better). Since computer simulations forecast the dependences upon the beam diameter and the beam shift [2], adjustments can be made while comparing the measured intensity profile with the calculated ones. The most important point with the laser mode is to confirm the quality (e.g. $M^{2}$ ) of the laser itself, and this should also be checked if beam vignetting or optics surface distortions occur during delivery of the beam. Although it is very difficult to adjust the modes of actual lasers, one method is to improve the mode quality with a spatial filter. It is in principle also possible to design a DOE for the measured intensity profile of the incoming laser beam, but there are some issues to be solved, including difficulties in measuring the phase distribution of the laser beam and in measuring the phase distribution of a mixed mode laser beam.

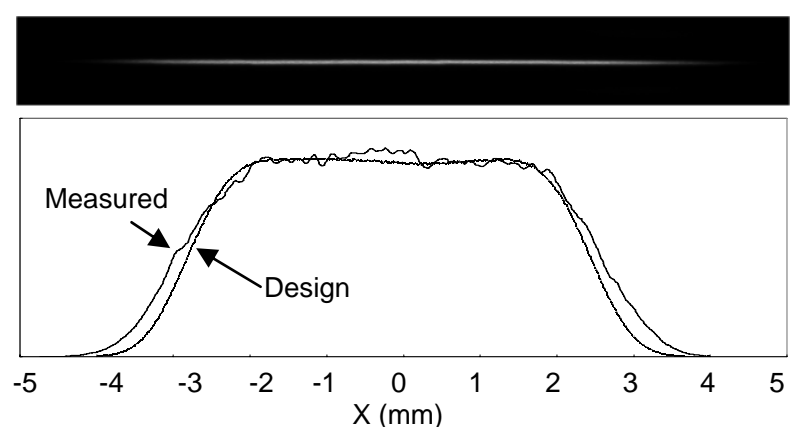

Fig. 7 Measured intensity profile of two line beam synthesis 


\section{Optics for multibeam processing}

Figure 8 shows the configuration of an optical system for multibeam laser processing. The system is configured with a diffractive beam splitter and $\mathrm{f}-\sin \theta$ lens which has $f \sin \theta$ distortion characteristics. While in conventional processing, a single laser beam is delivered and converged onto a spot on the surface of the workpiece, the diffractive element splits the single beam into multiple beams, and then the $f-\sin \theta$ lens converges them onto multiple spot locations that are required to be processed simultaneously. Process speed and throughput are improved according to the number of beams, and hence cost can be reduced. Replacing the splitter with others designed for different multibeam generation is effective in dealing with a wide variety of product specifications. Since two beam processing at a minimum is estimated to process twice as fast as a single beam, increasing the number of beams significantly increases the processing speed as long as the laser power is high enough for the requirements.

A diffractive beam splitter has a surface relief microstructure arranged periodically in a mosaic-like pattern. The periodic structure modulates the phase of the incident beam, which is usually flat or spherical, thereby functioning to split the single beam into multiple beams of specific directions and intensities. Diffraction gratings are the classical method, and have been popular in that they generate multiple-order diffraction beams. The DOE, however, has the advantages of being more flexible and having higher quality diffraction patterns than the gratings. It can produce a large number of beams, high uniformity for the peak intensities, and low noise intensities. The DOE is designed by computer-aided optimization of the surface complex structure, and is fabricated though microfabrication processes based on the production technology developed by the semiconductor industry, while the classical grating is designed analytically and its surface structure is mechanically ruled.

Let us start by considering diffraction through a simple grating that has multiple slits, before moving on to the DOE. The left side of Fig. 9 shows multiple slits with a period $\Lambda$, and a beam incident on the slits at an angle of $\theta_{\text {in }}$ with the $m$-th order beam outgoing from the silts at an angle of $\theta_{m}$. Here we choose two light rays, numbered 1 and 2 in the figure. Ray 1 passes position B and ray 2, another position $\mathrm{C}$. The distance between $\mathrm{B}$ and $\mathrm{C}$ is the

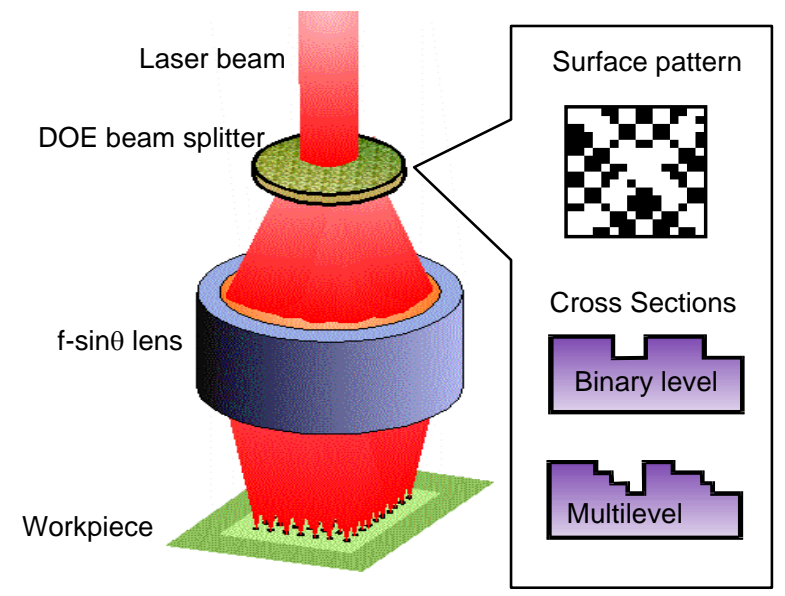

Fig. 8 Optical configuration for multibeam laser processing
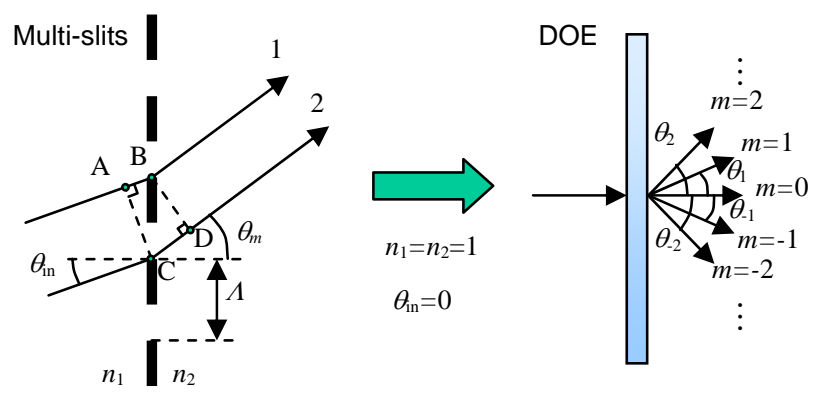

Fig. 9 Diffraction angles of multiple slits and DOE

same as the period $\Lambda$. The optical path difference $\Delta$ between rays 1 and 2 is given by:

$$
\Delta=\overline{\mathrm{CD}}-\overline{\mathrm{AB}}=\left(n_{2} \sin \theta_{m}-n_{1} \sin \theta_{i n}\right) \Lambda
$$

where $n_{1}$ and $n_{2}$ are the refractive indexes of the media on both sides of the slits. When $\Delta$ is equal to the product of the wavelength $\lambda$ and an integer $m$, which means there is no phase difference between rays 1 and 2 , the beam propagates in the direction $\theta_{m}$. This condition is represented by the following:

$$
n_{2} \sin \theta_{m}-n_{1} \sin \theta_{i n}=m \frac{\lambda}{\Lambda}
$$

where integer $m$ represent the $m$-th order diffraction. Equation (2) gives the diffraction angle corresponding to each order. With a periodic structure on the surface, the DOE determines the diffraction angles $\theta_{m}$ of the outgoing beams as well as the multiple slits. As shown on the right side of Fig. 9, assuming that the medium is air on both sides $\left(n_{1}=n_{2}=1\right)$, the incidence is perpendicular $\left(\theta_{\mathrm{in}}=0\right)$, and Eq. (2) can be simplified as follows:

$$
\sin \theta_{m}=m \frac{\lambda}{\Lambda}
$$

The above equation is the fundamental formula determining the directions of the beams from the DOE.

Since the DOE splitter described here has no function of a lens, the multiple beams are converged through a lens after leaving the DOE. Let us consider the optical configuration shown in Fig. 10, where the beam incident on the DOE is collimated, a converging lens with a focal length of $f$ is placed after the DOE, and only the zero-th and $m$-th diffraction beams are presented for simplicity. The location of the focus of the $m$-th beam on the focal plane, i.e., at a height $h_{m}$ from the optical axis, is represented by the following equations, depending on the distortion characteristics of the lens:

$$
h_{m}= \begin{cases}f \tan \theta_{m}=f \tan \sin ^{-1}\left(\frac{m \lambda}{\Lambda}\right) & \text { for an imaging lens } \\ f \theta_{m}=f \sin ^{-1}\left(\frac{m \lambda}{\Lambda}\right) & \text { for an } \mathrm{f} \theta \text { lens } \\ f \sin \theta_{m}=m \frac{\lambda f}{\Lambda} & \text { for an } \mathrm{f} \text {-sin } \theta \text { lens }\end{cases}
$$

A conventional imaging lens has an $f \tan \theta$ characteristic in order to make a conjugate image of the object without distortion, and an $f \theta$ lens used for scanning systems has literally $f \theta$ distortion. However, instead of those lenses, it is recommended to use an $f-\sin \theta$ lens for the diffractive beam splitters because of the $f \sin \theta$ distortion characteristics. This distortion involves the ratio of the height $h_{m}$ to the $m$ - 


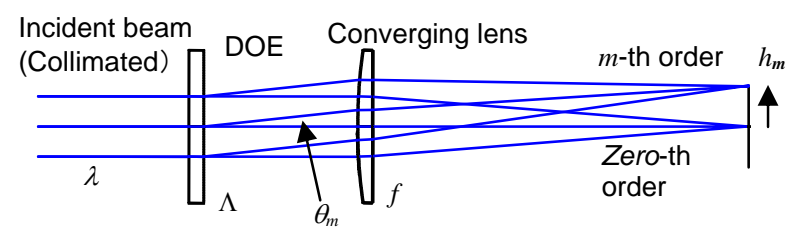

Fig. 10 Optical configuration of DOE and converging lens

th order diffraction, and then the spot spacing between neighboring beams $\Delta h=h_{m}-h_{m-1}$ is constant anywhere on the focal plane, being independent of $m$ :

$$
\Delta h=\frac{\lambda f}{\Lambda}
$$

Thus, the focal spots of the beams are equally pitched over the entire field of the $f-\sin \theta$ lens, and this means that when the diffraction beam has a large diffraction angle $\theta_{m}$ it affects the accuracy of the spot locations accordingly. For example, when $f=100 \mathrm{~mm}$ and $\theta_{m}=5.739 \mathrm{deg}$, the calculations of Eq. (4) give the values of $h_{m}$ for the three kinds of lenses:

$$
h_{m}=\left\{\begin{array}{l}
10.050 \mathrm{~mm} \text { for the imaging lens } \\
10.016 \mathrm{~mm} \text { for the } \mathrm{f} \theta \text { lens } \\
10.000 \mathrm{~mm} \text { for the } \mathrm{f}-\sin \theta \text { lens }
\end{array}\right.
$$

It is obvious that the spot location for the imaging lens differs by $50 \mu \mathrm{m}$ from that for the f-sin $\theta$ lens. Since drilling applications require less than $20-\mu \mathrm{m}$ positional accuracy including the accuracy of the motor-driven slide stages, the $50-\mu \mathrm{m}$ error caused by the lens is critical. In addition to the positional accuracy, the $f-\sin \theta$ lens is required to have a diffraction-limited focusing performance and image-space telecentricity over the entire field, even if the spots are widely spread with large spacing in the field. Hence it must be a multi-element lens and often has aspheric surfaces in order to reduce optical aberrations.

The period $\Lambda$ of the surface structure of the DOE determines the pitch of the spot arrangements with Eq. (5). Since the pitch is constant over the whole field, the spots are located on a regular grid pattern as shown in Fig. 11. In other words, the focal spots of the diffraction beams can never be placed freely in the field; their arrangements must, in principle, be limited to discrete coordinate positions

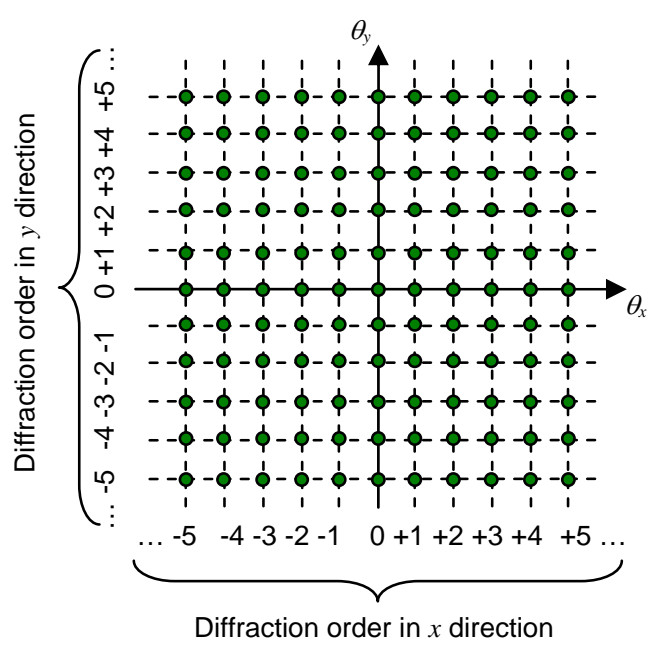

Fig. 11 Grid pattern representing spot arrangement represented by integral numbers of the diffraction orders. When the period $\Lambda$ increase as it doubles and triples, the pitch of the grid becomes smaller by one half and one third, respectively, and thus the flexibility of the spot locations is improved. However, it should be carefully noted that the finer pitch grid has many more crossing points of grid lines, and that noise possibly appears at all crossing points except points for signals. In order to avoid damage to the surface of the workpiece, noise intensities should be reduced through optimization of the surface structure of the DOE. But, they never become zero (usually less than $10 \%$ of the signal). Even if the noise intensity is low enough, when they come closer to the signals due to the narrower pitch of the grid, the feet of the beams are superposed on the signals, and then cause interference so that the uniformity of the signal intensities and the circularity of the spots may be degraded. Note that the pitch of the grid and the period of the DOE should thus be determined with great care.

While the diffraction angles are simply defined by Eq. (3) with the period and the wavelength, the intensity distribution of the diffracted beams is obtained in a more complex way, using a design method to define the microstructure within a period, i.e., a mosaic-like phase modulation pattern. Diffraction theory leads us to Fourier optics, where there is a specific relationship between the phase pattern and the reconstructed intensity distribution. Strictly speaking, the Fourier transform of the complex amplitude transmittance of the DOE yields a complex amplitude distribution on the reconstructed image [3]. At the optimization stage of DOE design, the fast Fourier transform (FFT) is iteratively calculated between the phase pattern and the image intensity, until obtaining a solution that achieves the required performance for diffraction efficiency, intensity uniformity and maximum noise intensity $[4,5]$. Optimization methods are categorized into two types, one of which minimizes the merit function, e.g. a direct binary search (DBS) [6], simulated annealing (SA), and genetic algorithm (GA), and the other of which does not utilize any merit functions, e.g. an iterative Fourier transform algorithm (IFTA) [7]. The former type has the advantage of balancing the intended optical properties, while IFTA is most suitable for optimizing large-scale patterns through faster calculations. Figure 12 shows a phase map (in gray scale) of the 16-phase level DOE splitter for the generation of $7 \times 7$ spot array for $\mathrm{CO}_{2}$ laser, which is designed by the DBS method [5]. That phase map represents the single period pattern that consists of $128 \mathrm{x}$ 128 square-cell array, and that is replicated like a tiled wall over the surface of the DOE. The optical properties of the DOE are $91.3 \%$ diffraction efficiency, $1.1 \%$ uniformity and

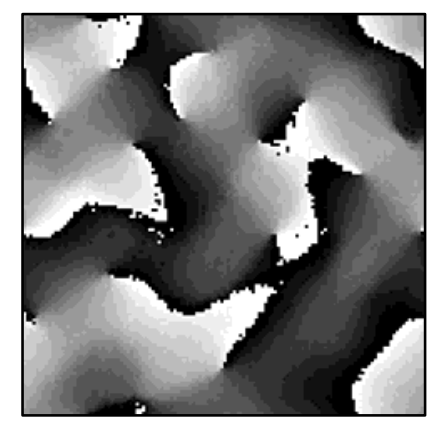

Fig. 12. Phase map of 16-level phase DOE for 7 x 7 spot array 


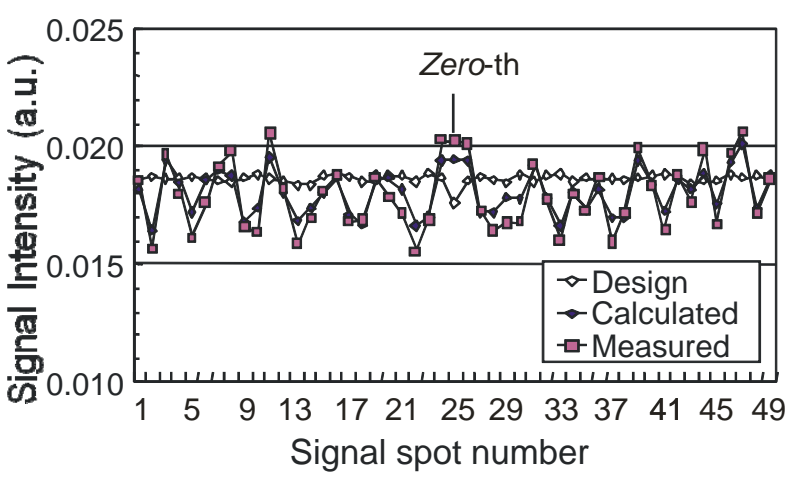

Fig. 13 Measured and calculated intensity uniformities of multilevel phase $7 \times 7$ spot array beam splitter

the noise intensity less than $3.1 \%$ of the signal average.

Either dividing the period into as many cells as possible or adopting a multilevel phase structure instead of a binary one are quite effective in increasing the degrees of freedom in the optimization. Accordingly, it gives us an increased diffraction efficiency, an improved intensity uniformity, and furthermore, a complex spot arrangement. Immoderate division into minute cells or easy adoption of a multiphase structure, however, tend to require too much accuracy in the microfabrication, and consequently an optimized performance cannot be obtained because it is too sensitive to fabrication errors. It is well known that the intensity of the zero-th order beam, which has a zerodegree diffraction angle and converges through the lens to a spot on the optical axis, changes dramatically due to fabrication errors $[5,8]$. Other order beams of the signals run into the problem of degradation of intensity uniformity [5]. That is why detailed tolerancing analyses are important and necessary in designing DOEs. Through drastic advances in computer technology, it has become possible to simulate a complex model, including errors in the DOE microfabrication, i.e., etching depth errors, width errors, mask alignment errors, etc. The simulation can accordingly predict the optical properties of the virtual DOE that is produced by the calculations. Figure 13 indicates a comparison between simulated and measured intensities of the 16-phase level DOE splitter shown in Fig. 12. This sample has rather large fabrication errors, so that the uniformity of the intensities does not appear to be very good. But the intensity variations due to fabrication errors indicate a good agreement between the simulation and the measurement.

A combination of a beam splitter and a homogenizer enables multispot flattop beam generation. Figure 14 shows an example of an optical system consisting of a diffractive homogenizer transforming an incoming beam into a flattop beam, a diffractive beam splitter splitting the flattop beam into multiple beams, and an $f-\sin \theta$ lens that makes an image of the multiple flattop beams on the image plane. Thus, the flattop beams simultaneously irradiate corresponding multiple spot locations on the workpiece. The rectangular mask in the figure is used to cut off noise around the flattop beam, which avoids any interference between the flattop beam and noise around the neighboring flattop beam on the image plane. The intensity uniformity of each split flattop beam is thereby improved. The $1.0 \mathrm{~mm}$ x $0.5 \mathrm{~mm}$ flattop beam generated by the previously described DOE homogenizer (Fig. 4) is split into three beams by the splitter, and then projected at $1 / 5$ magnification onto the image plane. The measured intensity profile is shown in Fig. 15, where the three 200 $\mu \mathrm{m} \times 100 \mu \mathrm{m}$ flattop beams are positioned with their size, uniformity and spacing of $300 \mu \mathrm{m}$ kept equal.

Finally, the author suggests some items which should be considered when applying multibeam processing to your applications.

i) The diffraction efficiency of the splitter is typically 50 to $70 \%$ for binary phase elements, and 70 to $90 \%$ for multilevel elements (this depends on the specifications of the spot array arrangements). A relatively large amount of laser power is scattered and lost as noise, so that the number of split beams should be determined by careful consideration of how much power your laser system has. Reasonably high intensity beams from a high-power laser should be shielded in order to maintain quality and safety control.

ii) Since the binary phase element has the real number amplitude (1 or -1$)$, the reconstructed image calculated with the FFT algorithm has a symmetric intensity distribution. The spot arrangement of the binary phase element is hence limited to being symmetric around the centered optical axis. An asymmetric arrangement is otherwise obtained with multilevel phase elements $(4,8$, 16 levels or more).

iii) The spot spacing is proportional to the wavelength $\lambda$, and is in inverse proportion to the period $\Lambda$ of the surface structure, as given by Eq. (5). Since a shorter wavelength makes it more difficult to increase the spot spacing, it is necessary to increase the focal length of the lens or to decrease the period. However, a long focal length is a tradeoff with small spot size for a fast lens having a low $\mathrm{F}$ number or a large numerical aperture. The small period imposes restrictions on the number of signal beams and the use of higher-order beams, and possibly causes problems with the fabrication accuracies as described previously.

iv) The spot spacing is highly dependent on the accuracy of the focal length of the lens and variations in the laser wavelength. High accuracy for the spacing requires some means of adjustment, i.e., a variable focal length for the lens, adjustable distance between the DOE splitter and

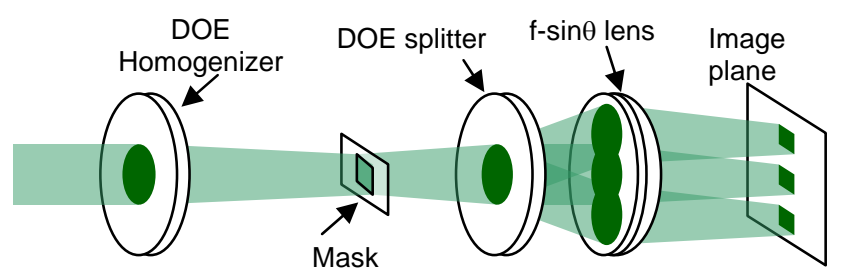

Fig. 14 Optical configuration for multispot flattop beams

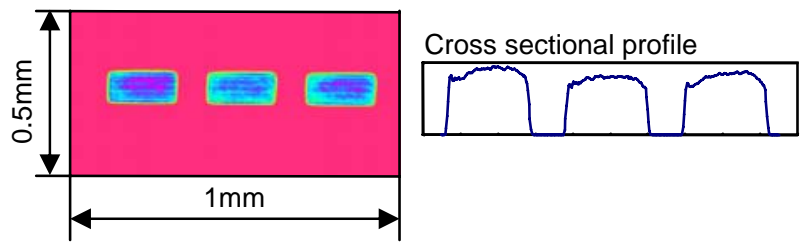

Fig. 15 Optical configuration for multispot flattop beams 
the lens, etc. It is also important to select a good laser system, because any fluctuations in the wavelength must be suppressed. Concerning the surface structure of the DOE, the period is still accurate since the fabrication process is similar to semiconductor devices, so that its influence on the spacing is usually negligible.

v) DOE beam splitters only split a beam into multiple beams, and thus have no effect on the propagation characteristics of the beams, except for the propagating direction. Uniform focusing characteristics can hence be obtained by using a good aberration corrected lens with off-axis aberrations reduced sufficiently.

vi) While a lateral shift in the incoming beam on the periodic structure of the DOE has no effect on beam splitting, any inclination between the separate axes of the DOE and the lens causes a distortion in the regular grid shown in Fig. 11. This distortion degrades the spot location accuracies, especially when setting a large pitch grid or using high-order diffraction beams, so careful adjustment is necessary when setting up both the DOE and the lens perpendicular to the optical axis.

\section{Conclusions}

The generation of various kinds of flattop beams and multibeam processing are achieved by employing aspheric and diffractive optics. Use of an $\mathrm{f}-\sin \theta$ lens is recommended to improve the positional accuracy of multiple spots. Further optical solutions are represented in this report for the synthesis of a long line flattop beam from independent laser beams and for simultaneous multispot flattop beam irradiation.

Consumer electronics companies have been making effort to fabricate high value-added products with their original processing technologies in order to compete in the worldwide cutthroat competition of the electronics industry.
Laser materials processing is a promising technology that is increasing its fields of application, since it features precision microfabrication, high accuracy and high controllability, low environmental load, etc., and hence is superior to conventional processes. In addition to solid state lasers, practical applications for optical components, units and systems will advance, and are expected to contribute to the future development of new materials and processes.

\section{References}

[1] K. Ebata, K. Fuse, T. Hirai and K. Kurisu: Proc. Fourth International Symposium on Laser Precision Microfabrication, Munich, SPIE Vol. 5063, (2003) p. 411.

[2] T. Hirai, K. Fuse, K. Kurisu, K. Ebata and K. Matsushima: Proc. Fifth International Symposium on Laser Precision Microfabrication, Nara, SPIE Vol. 5662, (2004) p. 378.

[3] J. W. Goodman: Introduction to Fourier Optics, (McGraw-Hill, New York, 1968).

[4] K. Fuse, K. Ebata, T. Okada, K. Kurisu and M. Shiozaki: Proc. High-Power Lasers in Manufacturing, Osaka, SPIE Vol.3888, (2000) p. 497.

[5] K. Fuse, T. Hirai, T. Ushiro, T. Okada, K. Kurisu and K. Ebata: J. Laser Appl., 15, (2003) 246.

[6] M. A. Seldowitz, J. P. Allebach, D. W. Sweeney: Appl. Opt., 26 (1987) 2788.

[7] J. Turunen, F. Wyrowski: Diffractive Optics for Industrial and Commercial Applications, (Akademie Verlag, Berlin, 1997).

[8] J. Amako, M. Murai and T. Sonehara: Opt. Rev., 5, (1998) 83.

(Received: July 13, 2009, Accepted: May 11, 2010) 